\title{
Gut Microbiota Changes in Inflammatory Bowel Diseases and Ankylosing Spondylitis
}

\author{
Anca Cardoneanu ${ }^{1}$, Cătălina Mihai ${ }^{2}$, Elena Rezus ${ }^{1}$, Alexandra Burlui ${ }^{1}$, Iolanda Popa ${ }^{2}$, Cristina Cijevschi Prelipcean ${ }^{2}$
}

1) Grigore T. Popa University

of Medicine and Pharmacy, Rehabilitation Clinical

Hospital, Iasi;

2) Grigore T. Popa University of Medicine and Pharmacy, Sf. Spiridon Emergency Hospital, Institute of Gastroenterology and Hepatology, Iasi, Romania

\begin{abstract}
Address for correspondence: Catalina Mihai Bd. Independentei no 1, Iasi, Romania, 700111; Email: catalinamihai@yahoo.com; ORCID: 0000-0001-9375-9224
\end{abstract}

Received: 17.07.2020

Accepted: 16.11.2020

\begin{abstract}
Background \& Aims: Both inflammatory bowel diseases (IBD) and ankylosing spondylitis (AS) can be considered chronic immune disorders sharing common etiopathogenetic mechanisms. Changes in the composition of the intestinal microbiota, which can lead to an abnormal mucosal response, could be the missing link between these two diseases. Our study evaluate the composition of intestinal microbiota and to characterize gut dysbiosis in patients with IBD and AS.

Methods: We conducted a prospective case-control study that enrolled 124 patients [20 Crohn's disease (CD), 27 ulcerative colitis (UC), 28 AS, 17 IBD + AS and 32 controls). Intestinal microbiota analysis was performed by real-time polymerase chain reaction in stool samples.

Results: The total quantity of bacteria was decreased in all investigated groups compared to the control group. In studied groups, we noticed an increased percentage of Bacteroides and Escherichia coli (E.coli) and a decreased percentage of Clostridium coccoides, Clostridium leptum, and Faecalibacterium prausnitzii compared to the control group. The percentages of Bifidobacterium $(\mathrm{p}=0.010)$ as well as Lactobacillus group $(\mathrm{p}=0.023)$ were higher in the L3 form of CD patients. In the E2 form of UC, the quantity of Bacteroides was much higher compared to the $\mathrm{E} 3$ form ( $\mathrm{p}=0.004$ ). In AS patients, significant correlations were observed only for the Bifidobacterium species, significantly increased in the axial form compared to peripheral disease $(\mathrm{p}=0.035)$. Statistically significant correlations were demonstrated between the Crohn Disease Activity Index score and the total bacterial group ( $\mathrm{p}=0.023, \mathrm{r}=-0.507)$, respectively Bacteroides $(\mathrm{p}=0.021, \mathrm{r}=-0.511)$ and between the Mayo score and Lactobacillus ( $\mathrm{p}=0.001)$, respectively E. coli $(\mathrm{p}=0.001)$. In IBD + AS group, the Crohn Disease Activity Index score was inversely correlated with the total bacterial group $(\mathrm{p}=0.010)$ and directly correlated with Lactobacillus ( $\mathrm{p}=0.047)$.

Conclusions: Intestinal dysbiosis is associated with both IBD and AS. In the association of IBD with AS, dysbiosis is intermediate, but it is associated with the more severe articular disease. Bifidobacterium and Lactobacillus (commonly used as probiotics!) were found to be increased in the association between active IBD and active AS. Further studies are needed to understand how dysbiosis regulates the gut immune system and contributes to intestinal and articular inflammation.
\end{abstract}

Key words: microbiota - inflammatory bowel disease - ankylosing spondylitis - gut dysbiosis.

Abbreviations: AS: ankylosing spondylitis; BASDAI: Bath Ankylosing Spondylitis Disease Activity Index; BASFI: Bath Ankylosing Spondylitis Functional Index; CD: Crohn's disease; CDAI: Crohn Disease Activity Index; C. coccoides: Clostridium coccoides (XIVa); C. leptum: Clostridium leptum (IV); E1: proctitis; E2: left colitis; E3: pancolitis; E. coli: Escherichia coli; F. prausnitzii: Faecalibacterium prausnitzii; IBD: inflammatory bowel diseases; L1: ileitis, L2: colitis; L3: ileocolitis; PCR: polymerase chain reaction; UC: ulcerative colitis.

\section{INTRODUCTION}

The intestinal microbiota comprises all the microorganisms in the microbial community belonging to the gastrointestinal tract. The concept of microbiome refers to a collective group of genomes including bacteria, bacteriophages, fungus, protozoa, and viruses. The study of gut microbiota is a current topic, and new techniques of microbiology and molecular biology have enabled the identification of the broad range of bacterial species involved. 
Currently, the gut microbiota composition and its functions have not been fully elucidated. It includes between 500 and 1000 bacterial species classified into four major categories from the phyla group: Bacteroidetes, Firmicutes, Proteobacteria, and Actinobacteria $[1,2]$.

Since birth, intestinal microbiota has an important role in the development of the intestinal immune system by modulating the formation of the intestinal mucosal layer and lymphoid structures, stimulating the immune system, differentiating the immune cells and producing immune mediators [3]. Changes in the intestinal microbiota composition can lead to dysbiosis, a decrease in gut microbial diversity and a modification of the balance between commensal and pathogenic microorganisms [4]. Intestinal dysbiosis is characterized by increased intestinal permeability and microbial translocation through the intestinal mucosa, which causes metabolic endotoxemia and systemic inflammation (increasing of proinflammatory cytokines and free oxygen radicals) [5]. Many studies have demonstrated the association between gut dysbiosis and various disorders such as nosocomial infections, premature necrotizing enterocolitis, inflammatory bowel diseases (IBD), irritable bowel syndrome, obesity, various autoimmune diseases or allergies [5-7].

Inflammatory bowel diseases and ankylosing spondylitis (AS) are chronic disorders sharing common etiopathogenetic mechanisms. Microscopic intestinal inflammation was found in $40-60 \%$ of AS patients [8]. Inflammatory bowel diseases can be considered the result of the interaction between genetic factors that determine susceptibility and environmental factors that influence the composition of the intestinal microbial flora, thus leading to an abnormal mucosal response [9]. The results of the published studies have demonstrated that IBD dysbiosis is characterized by a reduction in the diversity of bacterial species (especially anaerobic bacteria) followed by an increase in the concentration of entero-adherent bacteria [10-12]. On the other hand, in AS gut dysbiosis is also represented by a decreased total number of bacterial species, dominated by proinflammatory pathogens $[13,14]$. If gut dysbiosis is a cause or an effect of systemic inflammation or the missing link between these two diseases remains to be determined.

Building on all this published data, we aimed to evaluate the composition of intestinal microbiota and to characterize gut dysbiosis in patients having IBD [(Crohn's disease (CD), ulcerative colitis (UC)] or AS. Special attention was given to the intestinal microbiota analysis in patients who presented the association between IBD and AS.

\section{METHODS}

We conducted a prospective, case-control study in two academic centers in Northeastern Romania, one of gastroenterology and the other of rheumatology. The study enrolled 124 patients between April 2016 and March 2017. The control group comprised healthy people from the Rheumatology Clinic and the Rehabilitation Clinical Hospital in Iasi .

All included cases expressed their informed consent to participate in the study. The study was approved by the Ethics Committee of the University of Medicine and Pharmacy and the two hospitals from which patients were selected. All methods were carried out following the relevant local and international guidelines and regulations.

The inclusion criteria were: age over 18, the patient's signed informed consent, certain diagnoses of CD, UC, AS or association between IBD and AS. Diagnosis of IBD (CD or UC) was based on clinical symptoms, colonoscopy, and histopathology examination. The Montreal classification [15-17] was used to classify IBD by phenotype and to localize intestinal inflammation. Patients diagnosed with AS met the 1984 modified New York diagnostic criteria [18]. The subjects were asked to complete a questionnaire regarding dietary habits and antibiotic or probiotic use in the last three months. Exclusion criteria consisted of patient refusal to participate in this study, uncertain diagnosis of CD, UC or AS, colorectal cancer, serious infections (tuberculosis, Clostridium difficile), other comorbidities, use of antibiotics, probiotics, or restrictive diets in the last 3 months.

The enrolled cases were distributed as follows: (1) the group with CD - 20 cases, (2) the group with UC - 27 cases, (3) the group with AS - 28 cases, (4) the group with IBD and AS - 17 cases and (5) the control group - 32 cases.

Crohn's disease patients were divided into L1-ileitis, L2colitis, and L3-ileocolitis. UC patients were divided into E1proctitis, E2-left colitis, and E3-pancolitis and AS patients were grouped in axial disease and peripheral disease. For evaluating disease activity, Crohn Disease Activity Index (CDAI) scores were calculated for patients with $\mathrm{CD}$, the Mayo score was used for UC cases, and the Bath Ankylosing Spondylitis Disease Activity Index (BASDAI) and Bath Ankylosing Spondylitis Functional Index (BASFI) scores were used for AS cases [18]. Active CD was defined by a CD activity index of 150 and, respectively, active UC was defined by a UC activity index of $3[15,16]$. Intestinal microbiota analysis was performed using real-time polymerase chain reaction (real-time PCR) in the stool samples. From each patient included in the study, $20 \mathrm{~g}$ of faeces were obtained. The stool samples were transported as quickly as possible (within the same day) to the microbiology laboratory and were frozen at a temperature of $-80^{\circ}$ Celsius for a maximum of one week until DNA extraction.

For DNA extraction from feces, the GenEluteTM Stool DNA Isolation Kit, Sigma Aldrich, was used. DNA extraction included the following steps: (1) from the $20 \mathrm{~g}$ of faeces collected from patients, $200 \mathrm{mg}$ were isolated and added to a special extraction tube (Bead Tube) along with $1 \mathrm{ml}$ of Lysis Buffer $\mathrm{L}$, (2) $100 \mu \mathrm{L}$ of another special lysis solution (Lysis Additive A) was added, (3) mixed for 3 minutes, then centrifuged for 2 minutes at 14,000 rpm, (4) from the obtained supernatant, $600 \mu \mathrm{L}$ was transferred to another DNA tube (DNAase-free microcentrifuge tube) over which $100 \mu \mathrm{L}$ of Binding Buffer I was added and the mixture was incubated for 10 minutes on ice, then centrifuged for 2 minutes; (5) from the newly obtained supernatant, $700 \mu \mathrm{L}$ were separated in a $2 \mathrm{~mL}$ tube (DNAasefree microcentrifuge tube) over which $700 \mu \mathrm{L}$ of ethanol was added and centrifuged; (6) from the ethanol clarification supernatant, $600 \mu \mathrm{L}$ were separated, introduced into a specific DNA binding tube and centrifuged for 1 minute at $6000 \mathrm{rpm}$; (7) the DNA-binding column was mixed with $500 \mu \mathrm{L}$ of wash buffer (SK buffer) and centrifuged for 1 minute; (8) the washed DNA binding column was introduced into an Elution tube and 
$50 \mu$ of Elution Buffer (E) was added, centrifuged for 2 minutes at $2000 \mathrm{rpm}$, then 1 minute at $14.000 \mathrm{rpm}$.

After DNA extraction from the feces, DNA quantity and purity were checked using a NanoDrop spectrophotometer.

The PCR reaction targeted the following bacteria: total bacteria, Bacteroides, Bifidobacterium, Clostridium coccoides (XIVa) (C. coccoides), Clostridium leptum (IV) (C. leptum), Faecalibacterium prausnitzii (F. prausnitzii), Lactobacillus, Escherichia coli (E. coli). The $\beta$-globin gene was the internal control. The primer's structures were taken from the article published by Wang et al. [19] and verified using OligoAnalyzer 3.1 (https://eu.idtdna.com). Also, the primer's annealing temperature was checked.

The PCR reaction was performed using SYBR Green intercalary fluorochromes that only bind to double-stranded DNA molecules and included the following steps: (1) the amplification reaction was carried out in a final volume of $25 \mu \mathrm{L}$ containing: $9.8 \mu \mathrm{L}$ SYBR mix, $0.5 \mu \mathrm{L}$ of each primer at a final concentration of $0.2 \mu \mathrm{M}, 0.5 \mu \mathrm{L}$ fluorochrome ROX (5-carboxy-X-rhodamine), $5 \mu \mathrm{L}$ of bacterial DNA and ultrapure water to a volume of $20 \mu \mathrm{L}(9.8 \mu \mathrm{L}) ;(2) 1$ cycle at $95^{\circ} \mathrm{C}$ for 10 minutes; (3) 40 cycles at $95^{\circ} \mathrm{C}$ for 10 seconds; (4) 30 seconds at normalization temperature; (5) 30 seconds at $72^{\circ} \mathrm{C}$ - annealing temperature.

In order to reduce the quantitative error of detected bacteria and to characterize changes in bacterial copies, the abundance of the 16S rRNA gene was calculated from standard curves. Specific bacterial groups were expressed as a percentage of total bacteria identified using universal primers. The standard curve was constructed from decimal dilutions of the 16S rRNA amplicon using reference strains for each bacterial target. All sample amplification was performed based on this standard curve (Fig.1A). The dissociation and amplification curves confirmed that DNA amplification occurred specifically (Figs.1B, 1C, 1D).

The obtained data were centralized in the SPSS 22.0 database. Statistical analysis used both descriptive and analytical methods at 95\% significance level (CI 95\%). Some of the statistical methods used were: ANOVA and chi-square tests, linear regression, Odds Ratio. For comparisons between groups having a non-linear distribution, the Mann-Whitney U test and Kruskal-Wallis method were used. A p-value of less than 0.05 was considered statistically significant.

\section{RESULTS}

The characteristics of the patients included in our study are presented in Table I.

The total bacteria was lower in all studied groups (CD, UC, AS, IBD+AS) compared to the control group. The lowest intestinal microbiota was highlighted in patients with $C D$, followed by IBD + AS cases (Fig. 2). Patients with UC showed a higher total bacteria compared to $\mathrm{CD}$ and AS patients.

Specific bacterial counts were calculated as a percentage of the total bacterial counts of each study group. The diversity of intestinal microbiota for each study group is presented in Fig. 3. UC and CD had a similar profile, with an increased percentage of Bacteroides and E. coli and a decreased percentage of C. coccoides, C. leptum and F. prausnitzii compared to the control group. Patients with AS had similarities with the control group, while patients with associated IBD and AS seemed to have a similar bacterial distribution with IBD patients, with the highest percentage of Bacteroides from all studied groups.

In patients with $\mathrm{CD}$, statistically significant data regarding the location of the disease was observed only for total bacteria, Bifidobacterium, and Lactobacillus. The number of total bacteria was significantly increased in the L2 form of CD $(\mathrm{p}=0.034)$, while the percentages of Bifidobacterium $(\mathrm{p}=0.010)$ as well as Lactobacillus group ( $\mathrm{p}=0.023$ ) were higher in the L3 form of CD (Table II). In UC cases, significant correlations were highlighted only for Bacteroides. In the E2 form of UC, the quantity of Bacteroides was much higher compared to the E3 form $(\mathrm{p}=0.004)$. Other bacterial groups also presented a numerical growth in the E2 form of UC (Table III).

Concerning AS patients, significant correlations were observed only for the Bifidobacterium species, significantly increased in the axial form compared to peripheral disease $(\mathrm{p}=0.035)$. Many other bacterial groups were numerically increased in the axial form of AS (except Bacteroides), but without significant differences.

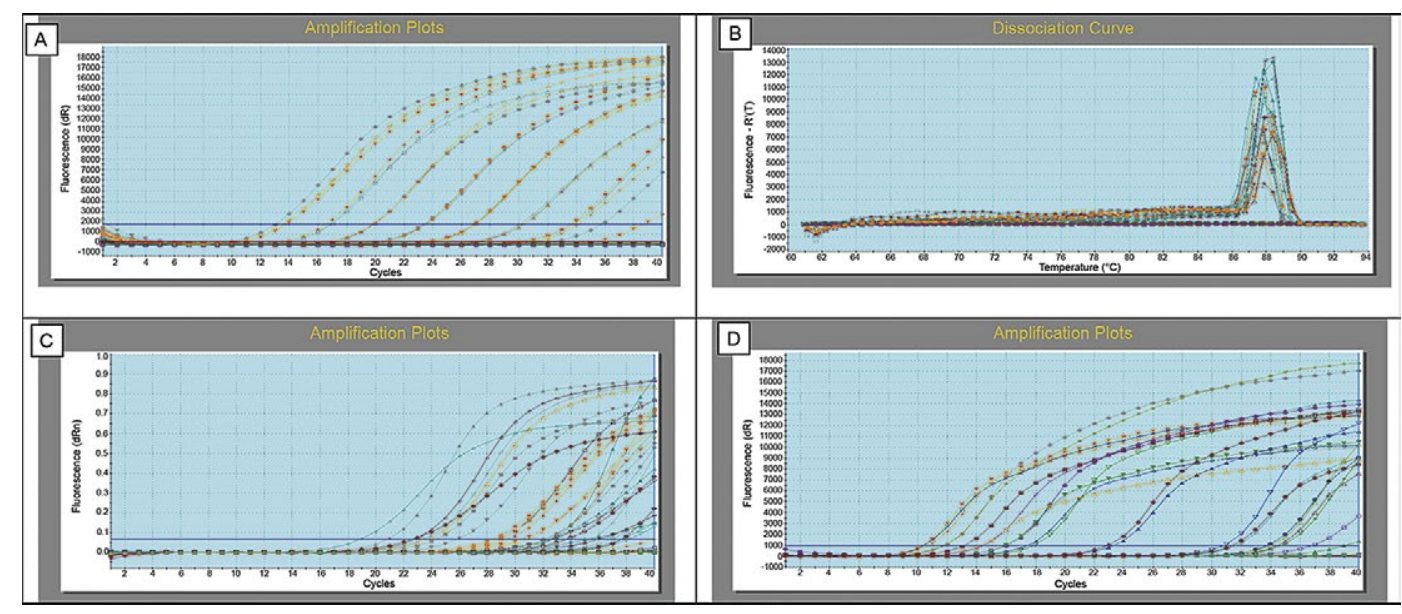

Fig.1. The dissociation and amplification curves for DNA amplification. A: The standard curve of amplification (triplicates); B Dissociation curve; C Amplification curve for E. coli; D Amplification curve for Bacteroides 
Table I. The characteristics of the study group

\begin{tabular}{|c|c|c|c|c|c|}
\hline & $\mathrm{CD}(\mathrm{n}=20)$ & $\mathrm{UC}(\mathrm{n}=27)$ & AS $(n=28)$ & $\begin{array}{c}\mathrm{IBD}+\mathrm{AS} \\
(\mathrm{n}=17)\end{array}$ & $\begin{array}{l}\text { Control } \\
(\mathrm{n}=32)\end{array}$ \\
\hline \multicolumn{6}{|l|}{ Gender (n,\%) } \\
\hline Female & $11(55 \%)$ & $12(44.4 \%)$ & $11(39.3 \%)$ & $5(29.4 \%)$ & $20(62.5 \%)$ \\
\hline Male & $9(45 \%)$ & $15(55.6 \%)$ & $17(60.7 \%)$ & $12(70.6 \%)$ & $12(37.5 \%)$ \\
\hline \multicolumn{6}{|l|}{ Age, years } \\
\hline Median (SD) & 51.2 & $48.93(9,7)$ & $52.1(13,6)$ & $52.4(8,9)$ & $61.5(10)$ \\
\hline Range & $46-55$ & $45-52$ & $46-57$ & $47-57$ & $57-65$ \\
\hline \multicolumn{6}{|l|}{ IBD extension (n) } \\
\hline E1 & NA & 5 & NA & NA & NA \\
\hline E2 & NA & 12 & NA & 8 & NA \\
\hline E3 & NA & 10 & NA & NA & NA \\
\hline $\mathrm{L} 1$ & 3 & NA & NA & NA & NA \\
\hline $\mathrm{L} 2$ & 6 & NA & NA & 5 & NA \\
\hline L3 & 11 & NA & NA & 4 & NA \\
\hline \multicolumn{6}{|l|}{ Disease activity } \\
\hline CDAI (Median) & 251.47 & NA & NA & 159.11 & NA \\
\hline Mayo (Median) & NA & 5.88 & NA & 4.37 & NA \\
\hline BASDAI (Median) & NA & NA & 4.83 & 4.01 & NA \\
\hline BASFI (Median) & NA & NA & 9.11 & 4.34 & NA \\
\hline
\end{tabular}

CD: Crohn's disease; UC: ulcerative colitis; AS: ankylosing spondylitis; IBD: inflammatory bowel disease; SD: standard deviation; E1: proctitis; E2: left colitis; E3: pancolitis; L1: ileitis, L2: colitis; L3: ileocolitis; CDAI: Crohn's Disease Activity Index; BASDAI: Bath Ankylosing Spondylitis Disease Activity Index; BASFI: Bath Ankylosing Spondylitis Functional Index; NA: not assessed.

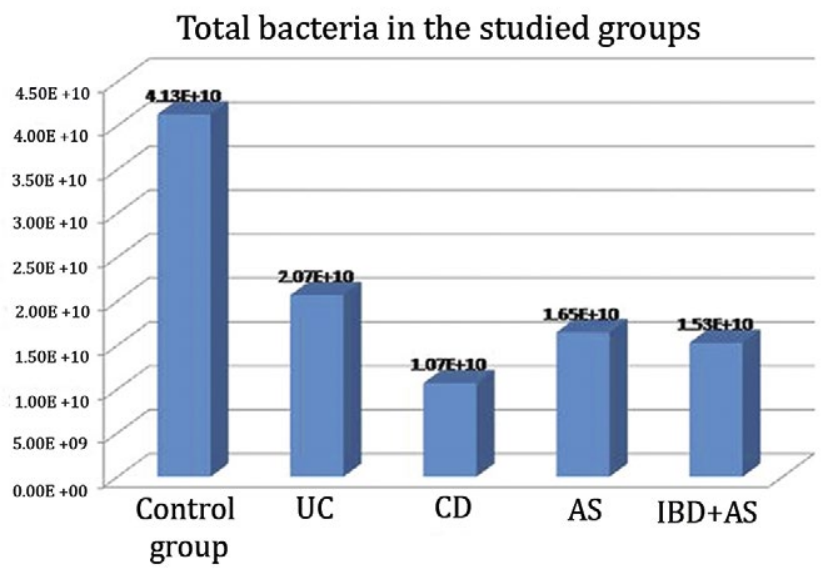

Fig. 2. Quantification of total bacteria in studied groups - log10CFU (faeces/g); CD: Crohn's disease; UC: ulcerative colitis; AS:ankylosing spondylitis; IBD: inflammatory bowel disease.

Statistically significant inverse correlations were demonstrated between the CDAI score and the total bacterial group ( $\mathrm{p}=0.023, \mathrm{r}=-0.507)$, respectively Bacteroides $(\mathrm{p}=0.021$, $\mathrm{r}=-0.511$ ). Positive Spearman correlations (but without statistical significance) were found between CDAI score and C. coccoides, Bifidobacterium and Lactobacillus. Significant associations were observed between the Mayo score and Lactobacillus ( $\mathrm{p}=0.001)$, respectively E. coli $(\mathrm{p}=0.001)$. In the group of AS cases, no significant correlations were observed between BASDAI and BASFI and the bacterial groups. However, significant associations have been demonstrated in IBD+AS patients. Thus, the BASDAI score was inversely correlated with the total bacterial group $(\mathrm{p}=0.010, \mathrm{r}=-0.606)$. In addition, the BASFI score correlated with all bacteria $(\mathrm{p}=0.001, \mathrm{r}=-0.764)$, F. prausnitzii $(\mathrm{p}=0.010, \mathrm{r}=0.606)$, Bifidobacterium $(\mathrm{p}=0.016, \mathrm{r}$ $=0.575)$, Lactobacillus $(\mathrm{p}=0,001, \mathrm{r}=0,843)$ and E. coli $(\mathrm{p}=0.016$, $\mathrm{r}=0.575)$. Table IV shows the correlations between intestinal microbiota composition and disease activity scores.

\section{DISCUSSIONS}

This is the first Romanian study that analyzes the composition of intestinal microbiota, using faeces real-time PCR, in patients with IBD, AS, and IBD associated with AS. The PCR reaction targeted the following bacteria: total bacteria, Bacteroides, Bifidobacterium, C. coccoides, C. leptum, F. prausnitzii, Lactobacillus, E. coli . The primer's structures were taken from the article published by Wang et al. [19] and verified using OligoAnalyzer 3.1 (https://eu.idtdna. com). Our analysis focused on the main bacteria found in the microbiome from all the main phyla: Firmicutes (Clostridiales, F. prausnitzii, Lactobacillus), Bacteroidetes (Bacteroides), Actinobacteria (Bifidobacterium), and Proteobacteria (E. coli). The main disadvantage is that many other species involved in IBD and/or AS gut dysbiosis (e.g. Ruminococcus, Prevotella, Faecalibacterium, etc) were not analyzed.

The total bacteria was lower in all investigated cases compared to the control group. Corroborating the presented results, we can divide the studied groups into 3 categories according to the importance of intestinal dysbiosis: groups with a 


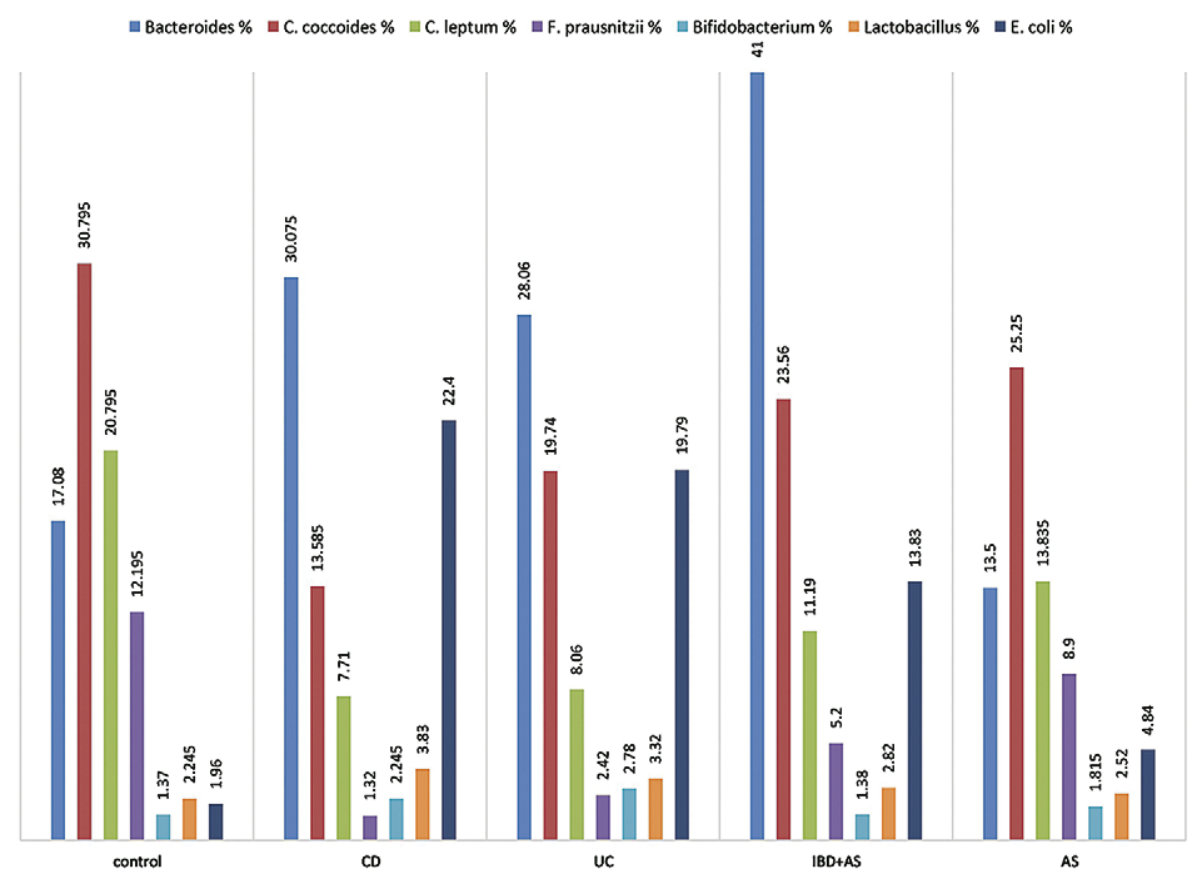

Fig. 3. Specific bacterial counts calculated as a percentage of the total bacterial counts of each study group. Crohn's disease, A: ulcerative colitis, B: ankylosing spondylitis, C: inflammatory bowel disease (CD or UC) + ankylosing spondylitis, D: control group.

Table II. Correlations between Crohn's disease location (L) and the analyzed microbial group

\begin{tabular}{lcccc}
\hline Bacteria & CD & N & Medium rank & P \\
\hline Total bacteria & L2 & 11 & 17.18 & 0.034 \\
Bacteroides \% & L3 & 15 & 10.80 & \\
& L2 & 11 & 16.91 & 0.05 \\
C. coccoides \% & L3 & 15 & 11.00 & \\
& L2 & 11 & 13.45 & 0.979 \\
C. leptum \% & L3 & 15 & 13.53 & \\
& L2 & 11 & 12.91 & 0.734 \\
F. prausnitzii \% & L3 & 15 & 13.93 & \\
& L2 & 11 & 14.45 & 0.582 \\
Bifidobacterium \% & L3 & 15 & 12.80 & \\
& L2 & 11 & 9.00 & 0.010 \\
Lactobacillus \% & L3 & 15 & 16.80 & \\
& L2 & 11 & 9.55 & 0.023 \\
E. coli \% & L3 & 15 & 16.40 & \\
& L2 & 11 & 10.18 & 0.056 \\
& L3 & 15 & 15.93 & \\
\hline
\end{tabular}

L: Crohn's disease location; L2: colonic; L3=ileo-colonic (L1= ileal was eliminated from statistical analysis - there were only 3 cases with constant values) C. coccoides: Clostridium coccoides (XIVa); C. leptum: Clostridium leptum (IV); E. coli: Escherichia coli; F. prausnitzii: Faecalibacterium prausnitzii.

significant dysbiosis: $\mathrm{CD}$ and $\mathrm{UC}$, the group with an intermediate dysbiosis: IBD associated with AS, and the low dysbiosis group: patients with AS. The link between the pathological gut and joint inflammation is not fully understood. Recent studies identified genes that encode proteins involved in the IL-23/Th 17 T-cell differentiation in both IBD and AS patients [20].
Table III. Correlations between the ulcerative colitis extension and analyzed microbial group

\begin{tabular}{|c|c|c|c|c|}
\hline Bacterial group & UC form & $\mathrm{N}$ & $\begin{array}{c}\text { Medium } \\
\text { Rank }\end{array}$ & $\mathrm{P}$ \\
\hline \multirow[t]{3}{*}{ Total bacteria } & E1 & 5 & 22.40 & 0.582 \\
\hline & E2 & 20 & 17.25 & \\
\hline & E3 & 10 & 17.30 & \\
\hline \multirow[t]{3}{*}{ Bacteroides \% } & E1 & 5 & 18.60 & 0.004 \\
\hline & E2 & 20 & 22.30 & \\
\hline & E3 & 10 & 9.10 & \\
\hline \multirow[t]{3}{*}{ C. coccoides $\%$} & E1 & 5 & 18.80 & 0.414 \\
\hline & E2 & 20 & 19.60 & \\
\hline & E3 & 10 & 14.40 & \\
\hline \multirow[t]{3}{*}{ C. leptum \% } & E1 & 5 & 14.80 & 0.097 \\
\hline & E2 & 20 & 21.20 & \\
\hline & E3 & 10 & 13.20 & \\
\hline \multirow[t]{3}{*}{ F. prausnitzii \% } & E1 & 5 & 19.00 & 0.268 \\
\hline & E2 & 20 & 19.95 & \\
\hline & E3 & 10 & 13.60 & \\
\hline \multirow[t]{3}{*}{ Bifidobacterium \% } & E1 & 5 & 14.00 & 0.344 \\
\hline & E2 & 20 & 17.20 & \\
\hline & E3 & 10 & 21.60 & \\
\hline \multirow[t]{3}{*}{ Lactobacillus \% } & E1 & 5 & 22.20 & 0.607 \\
\hline & E2 & 20 & 17.45 & \\
\hline & E3 & 10 & 17.00 & \\
\hline \multirow[t]{3}{*}{ E. coli \% } & E1 & 5 & 14.00 & 0.613 \\
\hline & E2 & 20 & 19.05 & \\
\hline & E3 & 10 & 17.90 & \\
\hline
\end{tabular}

E1: proctitis; E2: left colitis; E3: pancolitis. For abbreviations see Table II. 
Table IV. Correlations between the intestinal microbiota composition and disease activity scores

\begin{tabular}{|c|c|c|c|c|c|c|c|c|c|c|c|c|}
\hline & \multicolumn{2}{|c|}{ CDAI (CD group) } & \multicolumn{2}{|c|}{ MAYO (UC group) } & \multicolumn{2}{|c|}{ BASDAI (AS group) } & \multicolumn{2}{|c|}{$\begin{array}{l}\text { BASDAI (IBD+AS } \\
\text { group) }\end{array}$} & \multicolumn{2}{|c|}{ BASFI (AS group) } & \multicolumn{2}{|c|}{$\begin{array}{c}\text { BASFI (IBD+AS } \\
\text { group) }\end{array}$} \\
\hline & $\mathrm{r}$ & $\mathrm{p}$ & $\mathrm{r}$ & $\mathrm{p}$ & $\mathrm{r}$ & $\mathrm{p}$ & $\mathrm{r}$ & $\mathrm{p}$ & $\mathrm{r}$ & $\mathrm{p}$ & $\mathrm{r}$ & $\mathrm{p}$ \\
\hline All bacteria & -0.507 & 0.023 & -0.061 & 0.145 & -0.336 & 0.080 & -0.606 & 0.010 & -0.018 & 0.927 & -0.764 & 0.001 \\
\hline Bacteroides \% & -0.511 & 0.021 & 0.2 & 0.375 & -0.371 & 0.052 & -0.318 & 0.214 & -0.061 & 0.758 & -0.160 & 0.539 \\
\hline C. Coccoides \% & 0.198 & 0.402 & -0.239 & 0.90 & 0.111 & 0.573 & -0.265 & 0.304 & 0.053 & 0.788 & 0.050 & 0.849 \\
\hline C. leptum \% & -0.402 & 0.079 & -0.225 & 0.463 & 0.430 & 0.077 & -0.265 & 0.304 & 0.010 & 0.960 & 0.050 & 0.849 \\
\hline F. prausnitzii \% & -0.056 & 0.814 & -0.152 & 0.90 & -0.371 & 0.052 & 0.764 & 0.001 & 0.104 & 0.597 & 0.606 & 0.010 \\
\hline Bifidobacaterium \% & 0.171 & 0.471 & 0.182 & 0.118 & 0.183 & 0.351 & 0.063 & 0.810 & 0.048 & 0.809 & 0.575 & 0.016 \\
\hline Lactobacillus \% & 0.053 & 0.823 & 0.139 & 0.001 & 0.331 & 0.085 & 0.488 & 0.047 & 0.019 & 0.924 & 0.843 & 0.001 \\
\hline E. coli $\%$ & -0.01 & 0.96 & 0.156 & 0.001 & 0.309 & 0.110 & 0.063 & 0.810 & -0.01 & 0.941 & 0.575 & 0.016 \\
\hline
\end{tabular}

CDAI: Crohn's Disease Activity Index; BASDAI: Bath Ankylosing Spondylitis Disease Activity Index; BASFI: Bath Ankylosing Spondylitis Functional Index; CD: Crohn's disease; UC: ulcerative colitis; AS: ankylosing spondylitis; IBD: inflammatory bowel disease; r: Spearman correlation. For abbreviations see Table II

Gut T cells activated by antigens can migrate to the joints and induce inflammation. It has been shown that leukocytes populations from the inflamed gut can bind to synovial vessels and settle into the joint, using multiple adhesion molecules ( $\alpha \mathrm{E} \beta 7$ integrins, vascular adhesion protein-1, intracellular adhesion molecule-1 (ICAM-1/CD54) [21]. Our study suggests that other mechanisms, more than gut dysbiosis, could be involved in the pathogenesis of AS compared to IBD patients.

A quantitative and qualitative (biodiversity) reduction in gut microbiota in IBD patients is supported by numerous published studies [22-24]. The study published by Frank et al. [25] pointed out important changes in intestinal microbiota in patients with IBD. The authors noticed significant bacterial decreases especially in the phyla Firmicutes and Bacteroidetes, both in CD and UC cases. A reduction of the gut microbiome in IBD patients has been observed more than 15 years ago [26].

In IBD patients, both CD and UC, we noticed a decreased percentage of C. coccoides, C. leptum, and F. prausnitzii, followed by an increased percentage of Bacteroides and E. coli. F. prausnitzii produces butyrate and plays an important role in epithelial barrier integrity and immune modulation. A decrease in the C. leptum groups, especially $F$. prausnitzii, has been reported in many studies [27-31]. Regarding Bacteroides, the results of the studies are contradictory. Some of them reported a reduction of the Bacteroides group in IBD patients, especially in CD patients $[26,32,33]$ but others, similar to our results, demonstrated an increased amount of this phylum in IBD [23, $34,35]$. More than that, Said et al. [36] noticed an increase of Bacteroidetes phylum in salivary microbiota in UC patients. An increased percentage of $E$. coli, again similar to our results, has been demonstrated in many other studies $[23,37,38]$.

In patients with $\mathrm{CD}$, statistically significant data regarding the location of the disease were observed only for total bacteria, Bifidobacterium, and Lactobacillus. Many other studies demonstrated an increase of Bifidobacterium genera in IBD patients, notably in patients with $\mathrm{CD}$, especially in the ileal location [11, 19, 23, 39]. In UC cases, significant correlations were highlighted only for Bacteroides. It could be inferred that, in CD patients, the extension of intestinal inflammation is a significant factor for intestinal dysbiosis. Our results are consistent with the data published in the literature. The study of Vrakas et al. [40] confirms the worsening of intestinal dysbiosis in an extended and active form of CD. On the other hand, in UC cases, most bacterial groups showed a numerical growth in E2 form. The results of different studies are contradictory. Some support the worsening of intestinal dysbiosis in patients with an extensive and active UC [40]. However, the Pascal et al. study [41] presents the augmentation of dysbiosis in proctitis. Many factors may explain these discrepancies: sample source (biopsy or stool), disease activity (flare or remission), medication, comorbidities, diet, smoking status, body mass index, methods used to analyze the microbiota, etc [42].

The number of studies regarding intestinal microbiota analysis in AS patients is lower, but they also support the presence of intestinal dysbiosis characterized by a decrease in total bacterial diversity [43, 44]. In 2017 Wen et al. [45] published an article that highlighted the particular microbial profile in AS patients. In this study, the AS patients showed increases in the amount of Prevotella melaninogenica, Prevotella copri, and Prevotella sp. C561 and decreases in Bacteroides spp. Similar to our results, Bifidobacterium was increased in AS patients [45]. Many other studies noticed a higher amount of the Bifidobacterium genus, including B. bifidum species in AS patients [46, 47]. Again similar to our results, Bacteroides genera were found to be higher in AS patients by several other studies $[47,48]$.

Our study also analyzed the composition of intestinal microbiota in patients with the association of IBD and AS. We found that the level of dysbiosis in IBD associated with AS was intermediate, less than in IBD and more than in AS. Compared with AS, we found an increase of Bacteroides and a decrease of F. prausnitzii, C. coccoides, C. leptum. Compared with IBD, we found an increase of Bacteroides and E.coli and a decrease of F. prausnitzii. Many other studies have demonstrated a decrease of Fecalibacterium in patients with both diseases (IBD and AS) [23, 32, 46]. Salem et al. meta-analysis [49] focused on similarities and differences in gut microbiome in patients with chronic rheumatic inflammatory diseases and IBD. The authors noticed an increase in Firmicutes genera Lactobacillus and Staphylococcus, Actinobacteria, Bifidobacterium, and Proteobacteria genera such as Pseudomonas, Klebsiella, and Proteus, whereas Firmicutes phyla, Faecalibacterium, Roseburia genera, and Verrucomicrobia phylum were decreased in both chronic rheumatic diseases and IBD. 
Last but not least, our study investigated the link between intestinal microbiota and the extent and activity of the diseases considered. Our results support the close relationship between disease activity and the degree of intestinal dysbiosis. The higher CDAI and Mayo scores are, the more important intestinal dysbiosis becomes. We observed significant associations between the Mayo score and Lactobacillus. Our results are similar to those of Wang et al. [19] who found that Bifidobacterium and the Lactobacillus group were increased in active IBD patients. In his study, Zhang et al. [50] demonstrated that, in UC, Lactobacilli were significantly different between the ulcerated and non-ulcerated regions.

In patients with AS, no significant correlations were observed between activity scores (BASDAI, BASFI) and the bacterial groups. Only for the group with AS and IBD association, there were significant correlations between the composition of gut microbiota and BASDAI, respectively BASFI scores. An increase in AS activity corroborated with a decrease in functionality can be associated with an increase in pro-inflammatory bacteria.

An interesting finding of our study is the unexpected significant correlation between the Lactobacillus and Bifidobacterium with UC activity, BASFI, and BASDAI scores in the IBD associated with AS group, and, respectively AS and BASFI scores in the combination group. Salem et al [49] noticed an increase in Lactobacillus and Bifidobacterium in both IBD and chronic rheumatic diseases. More than that, Lactobacillus R. Gnavus was more present in mice with arthritis and colitis compared with an arthritis - only group [51]. Further studies are necessary to determine if it is an epiphenomenon due to ecological niche competition or if there are some specific species involved or it is a microbial signature in IBD associated with AS. Until then we have to be more cautious when prescribing probiotics containing Lactobacilli and Bifidobacterium to patients with active IBD associated with active AS.

Our study has several limitations: it analyzed a small number of bacterial populations ( 3 species, 2 genera, 1 phylum) and therefore cannot sufficiently characterize the entire microbiota (all bacteria, but also virus or fungi); for microbiome analysis, we used RT-PCR, which is less specific than $16 \mathrm{~S}$ rRNA gene sequencing or shotgun metagenomics sequencing. Next generation sequencing or shotgun metagenomics are considered superior when compared to RT-PCR, with a higher power to detect novel genes and a higher sample throughput. However, RT-PCR remains a viable method, with a familiar workflow, and it has been successfully applied for quantification of bacterial DNA in many previous studies regarding microbiota changes. We analyzed only faeces samples, not colonic biopsies; we did not compare various treatments (immunomodulators, biologics) that can influence the composition of the microbiota. With all these limitations, as far as we know, it is the first study which directly compares the composition of gut microbiota in IBD, AS, and association IBD + AS patients.

\section{CONCLUSIONS}

Our results support the presence of a link between intestinal bacterial composition, IBD, and AS. Intestinal dysbiosis in patients with $\mathrm{CD}$ and $\mathrm{UC}$ is quite similar, being more pronounced compared to the control group or AS patients and it is correlated with disease activity. In the association of AS with IBD dysbiosis it is less significant compared to IBD, but it is associated with higher rheumatic disease activity scores. Bifidobacterium and Lactobacillus were found to be increased in the association between active IBD and active AS. Further studies are required for a better understanding of the interactions between the microbiome and immune system within the microbiota-joint-gut axis.

Conflicts of interest: None to declare.

Authors' contribution: A.C. drafted the manuscipt. A.C., C.C., A.B., I.P. collected and analyzed the data. E.R. revised the literature, critically revised the manuscript. C.C.P. conceived and designed the study and critically revised the manuscript.

Anknowledgements: This work was supported by the Grigore T. Popa University of Medicine and Pharmacy through decision no 19690 of 14.09.2015. The doctoral research funding for $\mathrm{PhD}$ students represented $35 \%$ of the $\mathrm{PhD}$ grant amount budgeted by the Ministry of Education and Scientific Research. The money was allocated for the purchase of laboratory supplies necessary for $\mathrm{PhD}$ research.

Supplementary material: To access the supplementary material visit the online version of the J Gastrointestin Liver Dis at http://dx.doi. org/10.15403/jgld-2823

\section{REFERENCES}

1. Guarner F, Malagelada JR. Gut flora in health and disease. Lancet 2003;361:512-519. doi:10.1016/S0140-6736(03)12489-0

2. Human Microbiome Project Consortium. Structure, function and diversity of the healthy human microbiome. Nature 2012;486:207-214. doi:10.1038/nature 11234

3. Akira S, Uematsu S, Takeuchi O. Pathogen recognition and innate immunity. Cell 2006;124:783-801. doi:10.1016/j.cell.2006.02.015

4. Ni J, Wu GD, Albenberg L, Tomov VT. Gut microbiota and IBD: causation or correlation? Nat Rev Gastroenterol Hepatol 2017;14:573584. doi:10.1038/nrgastro.2017.88

5. Melmed GY, Abreu MT. New insights in to the pathogenesis of inflammatory bowel disease. Curr Gastroenterol Rep 2004;6:474-481. doi:10.1007/s11894-004-0069-3

6. Li J, Butcher J, Mack D, Stintzi A. Functional impacts of the intestinal microbiome in the pathogenesis of inflammatory bowel disease. Inflamm Bowel Dis 2015;21:139-153. doi:10.1097/MIB.0000000000000215

7. Das B, Nair GB. Homeostasis and dysbiosis of the gut microbiome in health and disease. J Biosci 2019;44:117.

8. Van Praet L, Van den Bosch FE, Jacques P, et al. Microscopic gutinflammation in axial spondyloarthritis:a multiparametric predictive model. Ann Rheum Dis 2013;72:414-417. doi:10.1136/ annrheumdis-2012-202135

9. Molodecky NA, Kaplan GG. Environmental risk factors for inflammatory bowel disease. Gastroenterol Hepatol (NY) 2010;6:339-346.

10. Nishida A, Inoue R, Inatomi O, Bamba S, Naito Y, Andoh A. Gut microbiota in the pathogenesis of inflammatory bowel disease. Clin J Gastroenterol 2018;11:1-10. doi:10.1007/s12328-017-0813-5 
11. Khan I, Ullah N, Zha L, et al. Alteration of Gut Microbiota in Inflammatory Bowel Disease (IBD): Cause or Consequence? IBD Treatment Targeting the Gut Microbiome. Pathogens 2019;8:126. doi:10.3390/pathogens8030126

12. Walker AW, Sanderson JD, Churcher C, et al. High-throughput clone library analysis of the mucosa-associated microbiota reveals dysbiosis and differences between inflamed and noninflamed regions of the intestine in inflammatory bowel disease. BMC Microbiol 2011;11:7. doi:10.1186/1471-2180-11-7

13. Breban $M$, Beaufrère $M$, Glatigny $S$. The microbiome in spondyloarthritis. Best Pract Res Clin Rheumatol 2019;33:101495. doi:10.1016/j. berh.2020.101495

14. Costello ME, Ciccia F, Willner D, et al. Intestinal dysbiosis in ankylosing spondylitis. Arthritis Rheumatol 2015;67:686-691. doi:10.1002/ art.38967

15. Magro F, Gionchetti P, Eliakim R, et al. Third European Evidence-based Consensus on Diagnosis and Management of Ulcerative Colitis. Part 1: Definitions, Diagnosis, Extra-intestinal Manifestations, Pregnancy, Cancer Surveillance, Surgery, and Ileo-anal Pouch Disorders. J Crohns Colitis 2017;11:649-670. doi:10.1093/ecco-jcc/jjx008

16. Gomollón F, Dignass A, Annese V, et al. 3rd European Evidence-based Consensus on the Diagnosis and Management of Crohn's Disease 2016: Part 1: Diagnosis and Medical Management. J Crohns Colitis 2017;11:325. doi:10.1093/ecco-jcc/jjw168

17. Silverberg MS, Satsangi J, Ahmad T, et al. Toward an integrated clinical, molecular and serological classification of inflammatory bowel disease: report of a Working Party of the 2005 Montreal World Congress of Gastroenterology. Can J Gastroenterol 2005;19(Suppl A):5A-36A. doi:10.1155/2005/269076

18. van der Linden S, Valkenburg HA, Cats A. Evaluation of diagnostic criteria for ankylosing spondylitis. A proposal for modification of the New York criteria. Arthritis Rheum 1984;27:361-368. doi:10.1002/ art.1780270401

19. Wang W, Chen L, Zhou R, et al. Increased proportions of Bifido bacterium and the Lactobacillus groupandloss of butyrate-producing bacteria in inflammatory bowel disease. J Clin Microbiol 2014;52:398406. doi:10.1128/JCM.01500-13

20. Fragoulis GE, Siebert S, McInnes IB. Therapeutic Targeting of IL-17 and IL-23 Cytokines in Immune Mediated Diseases. Annu Rev Med 2016;67:337-353. doi:10.1146/annurev-med-051914-021944

21. Fragoulis GE, Liava C, Daoussis D, Akriviadis E, Garyfallos A, Dimitroulas T. Inflammatory bowel diseases and spondyloarthropathies: From pathogenesis to treatment. World J Gastroenterol 2019;25:2162 2176. doi:10.3748/wjg.v25.i18.2162

22. Willing B, Halfvarson J, Dicksved J, et al. Twin studies reveal specific imbalances in the mucosa-associated microbiota of patients with ilea Crohn's disease. Inflamm Bowel Dis 2009;15:653-660. doi:10.1002/ ibd. 20783

23. Willing BP, Dicksved J, Halfvarson J, et al. A pyrosequencing study in twins shows that gastrointestinal microbial profiles vary with inflammatory bowel disease phenotypes. Gastroenterology 2010;139:1844-1854.e1. doi:10.1053/j.gastro.2010.08.049

24. Wills ES, Jonkers DM, Savelkoul PH, Masclee AA, Pierik MJ, Penders J. Fecal Microbial Composition of Ulcerative Colitis and Crohn's Disease Patients in Remission and Subsequent Exacerbation. PLoS One 2014;9:e90981. doi:10.1371/journal.pone.0090981

25. Frank DN, St Amand AL, Feldman RA, Boedeker EC, Harpaz N, Pace NR. Molecular-phylogenetic characterization of microbial community imbalances in human inflammatory bowel diseases.
Proc Natl Acad Sci U S A 2007;104:13780-13785. doi:10.1073/ pnas.0706625104

26. Ott SJ, Musfeldt M, Wenderoth DF, et al. Reduction in diversity of the colonic mucosa associated bacterial microflora in patients with active inflammatory bowel disease. Gut 2004;53:685-693. doi:10.1136/ gut.2003.025403

27. Sokol H, Pigneur B, Watterlot L, et al. Faecalibacterium prausnitzi is an antiinflammatory commensal bacterium identified by gut microbiota analysis of Crohn's disease patients. Proc Natl Acad Sci U S A 2018;105:16731-16736. doi:10.1073/pnas.0804812105

28. Scanlan PD, Shanahan F, O’Mahony C, Marchesi JR. Cultureindependent analyses of temporal variation of the dominant fecal microbiota and targeted bacterial subgroups in Crohn's disease. J Clin Microbiol 2006;44:3980-3988. doi:10.1128/JCM.00312-06

29. Machiels K, Joossens M, Sabino J, et al. A decrease of the butyrateproducing species Roseburiahominis and Faecali bacterium prausnitzii defines dysbiosis in patients with ulcerative colitis. Gut 2014;63:12751283. doi:10.1136/gutjnl-2013-304833

30. Fujimoto T, Imaeda H, Takahashi K, et al. Decreased abundance of Faecali bacterium prausnitzii in the gut microbiota of Crohn's disease. J Gastroenterol Hepatol 2013;28:613-619. doi:10.1111/jgh.12073

31. Varela E, Manichanh C, Gallart M, et al. Colonisation by Faecali bacterium prausnitzii and maintenance of clinical remission in patients with ulcerative colitis. Aliment Pharmacol Ther 2013;38:151-161. doi:10.1111/apt.12365

32. Gevers D, Kugathasan S, Denson LA, et al. The treatment-naive microbiome in new-onset Crohn's disease. Cell Host Microbe 2014;15:382-392. doi:10.1016/j.chom.2014.02.005

33. Aomatsu T, Imaeda H, Fujimoto T, et al. Terminal restriction fragment length polymorphism analysis of the gut microbiota profiles of pediatric patients with inflammatory bowel disease. Digestion 2012;86:129-135. doi:10.1159/000339777

34. Andoh $\mathrm{A}$, Imaeda $\mathrm{H}$, Aomatsu $\mathrm{T}$, et al. Comparison of the fecal microbiota profiles between ulcerative colitis and Crohn's disease using terminal restriction fragment length polymorphism analysis. J Gastroenterol 2011;46:479-486. doi:10.1007/s00535-010-0368-4

35. Rehman A, Rausch P, Wang J, et al. Geographical patterns of the standing and active human gut microbiome in health and IBD. Gut 2016;65:238248. doi:10.1136/gutjnl-2014-308341

36. Said HS, Suda W, Nakagome S, et al. Dysbiosis of salivary microbiota in inflammatory bowel disease and its association with oral immunological biomarkers. DNA Res 2014;21:15-25. doi:10.1093/dnares/dst037

37. Duboc H, Rajca S, Rainteau D, et al. Connecting dysbiosis, bile-acid dysmetabolism and gut inflammation in inflammatory bowel diseases. Gut 201;62:531-539. doi:10.1136/gutjnl-2012-302578

38. Knoll RL, Forslund K, Kultima JR, et al. Gut microbiota differs between children with Inflammatory Bowel Disease and healthy siblings in taxonomic and functional composition: a metagenomic analysis. Am J Physiol Gastrointest Liver Physiol 2017;312:G327-G339. doi:10.1152/ ajpgi.00293.2016

39. Morgan XC, Tickle TL, Sokol H, et al. Dysfunction of the intestinal microbiome in inflammatory bowel disease and treatment. Genome Biol 2012;13:R79. doi:10.1186/gb-2012-13-9-r79

40. Vrakas S, Mountzouris KC, Michalopoulos G, et al. Intestinal Bacteria Composition and Translocation of Bacteria in Inflammatory Bowel Disease. PLoS One 2017;12:e0170034. doi:10.1371/journal. pone.0170034

41. Pascal V, Pozuelo M, Borruel N, et al. A microbial signature for Crohn's disease. Gut 2017;66:813-822. doi:10.1136/gutjnl-2016-313235 
42. Matsuoka K, Kanai T. The gut microbiota and inflammatory bowel disease. Semin Immunopathol 2015;37:47-55. doi:10.1007/s00281014-0454-4

43. Stebbings S, Munro K, Simon MA, et al. Comparison of the faecal microflora of patients with ankylosing spondylitis and controls using molecular methods of analysis. Rheumatology 2002;41:1395-1401. doi:10.1093/rheumatology/41.12.1395

44. Matzkies FG, Targan SR, Berel D, et al. Markers of intestinal inflammation in patients with ankylosing spondylitis: a pilot study. Arthritis Res Ther 2012;14:R261. doi:10.1186/ar4106

45. Wen C, Zheng Z, Shao T, et al. Quantitative metagenomics reveals unique gut microbiome biomarkers in ankylosing spondylitis. Genome Biol 2017;18:142. doi:10.1186/s13059-017-1271-6

46. Breban M, Tap J, Leboime A, et al. Faecal microbiota study reveals specific dysbiosis in spondyloarthritis. Ann Rheum Dis 2017;76:16141622. doi:10.1136/annrheumdis-2016-211064
47. Stoll ML, Weiss PF, Weiss JE, et al. Age and fecal microbial strainspecific differences in patients with spondyloarthritis. Arthritis Res Ther 2018;20:14. doi:10.1186/s13075-018-1510-6

48. Zhang $\mathrm{X}$, Zhang $\mathrm{D}$, Jia $\mathrm{H}$, et al. The oral and gut microbiomes are perturbed in rheumatoid arthritis and partly normalized after treatment. Nat Med 2015;21:895-905. doi:10.1038/nm.3914

49. Salem F, Kindt N, Marchesi JR, et al. Gut microbiome in chronic rheumatic and inflammatory bowel diseases: Similarities and differences. United European Gastroenterol J 2019;7:1008-1032. doi:10.1177/2050640619867555

50. Zhang M, Liu B, Zhang Y, Wei H, Lei Y, Zhao L. Structural shifts of mucosa-associated lactobacilli and Clostridium leptum subgroup in patients with ulcerative colitis. J Clin Microbiol 2007;45:496-500. doi:10.1128/JCM.01720-06

51. Liu X, Zeng B, Zhang J, et al. Role of the gut microbiome in modulating arthritis progression in mice. Sci Rep 2016;6:30594. doi:10.1038/srep30594 